\title{
The Innovative Research from the Angle of Public Problem on Governance Mechanism of Flight Delays
}

\author{
Liu Guangcai ${ }^{1,2}$, Chen jingjing ${ }^{1}$, Liu kexin ${ }^{3}$ \\ ${ }^{1}$ Economics and Management College, Civil Aviation University of China, Tianjin 300300, China \\ ${ }^{2}$ College of Management, Tianjin University, Tianjin 300071, China \\ ${ }^{3}$ Law school, Civil Aviation University of China, Tianjin 300300, China \\ lgc4201@ sohu.com, caucchenjingjing@sohu.com
}

\begin{abstract}
Flight delay is a public problem in civil aviation industry, and resolving the disputes arising from flight delay has been beyond the control of airlines. To solve the problem, it needs the government and the private sector to work together. In this paper, by analyzing the theory of public issues, we proposed to set sectoral governance institutions of flight delays and introduce the third party governance corporation of flight delays. As complementary measures of the proposed ideas, we also designed the process and mechanism of economic compensation reserve fund of flight delays.

Index Terms - governance mechanism of flight delays, sectoral governance institutions of flight delays, governance corporation of flight delays, economic compensation reserve fund of flight delays

Flight delay is a public problem in civil aviation industry, there are flight delays in each country, more or less. As the same as the Civil Aviation developed countries, the delay rate in China is generally around $20 \%$. However, the flight delay in China, which is characterized by high frequency, long-standing, disputes, contradictions intense, has become a public issues in civil aviation industry. Rely solely on airlines, it is difficult to really solve the dispute and mechanism innovation must be carried out[1]. In this paper, by analyzing the theory of public issues, we proposed to set sectoral governance institutions of flight delays or introduce the third party governance corporation of flight delays. As complementary measures of the proposed ideas, we also designed the process and mechanism of economic compensation reserve fund of flight delays.
\end{abstract}

\section{The Public Problems and Related Theories}

Public issues is the core of public administration, refers to the public problem that related to the members of society, life and other vital interests, affect all or most of the members of the society's normal life and even make social progress distorted.

There are three basic characteristics of public issues:(1)extensive influence;(2)the urgency of using social forces or resources to solve such problems;(3)developing and changing.

According to the affected scope of the public issues, they can be divided into global, national, regional, and communitybased public issues. Different levels of public problems belong to different management body. According to the nature of the problem, there are four categories of the public issues: regulatory problems, infrastructure problems, service and social security problems. Generally, the first three are managed by the power sector, public sector and social behavior, while service problems can be solved by government and private sector.[2]

According to the new public management theory, service problems can be solved by the government, but the efficiency of the administrative governance model is generally low. Therefore, the new management model should take the efficiency as the center be market-oriented. It is also necessary to introduce private-sector or third-party social forces to improve management efficiency.

\section{Flight Delays are Industry-Specific Public Issues}

Flight delays should be solved by airlines themselves, for the reason that flight delays is essentially a service failure. However, as the conflict between airlines and passengers caused by the flight delays is so prominent, this problem in China should be considered as public issues. The reasons for this are as follows:

(1)In China, the total amount of delayed flights is large, and the influence is wide.

The rate of Civil Aviation Flight delays in China has fallen to below $20 \%$ since Reform and Opening-up. However, with the great development of air transport and the rapid growth of flights, the absolute number of delayed fights is in a sharp rise. In 2000, the delayed flights was 142,700 sorties, and the number in 2008 was increased to 260,000 sorties. Each year, about $1 / 5$ of travelers suffered flight delays and there were nearly 40 millions passengers suffered flight delays. According to the data of 2004, the losses to the United States, European airlines caused by flight delays were up to 6.2 billion, 5 billion dollar. In China, there is also an annual loss of millions of dollars caused by flight delays.[3] Consequently, the impact of flight delays is widely and far-reaching.

(2)The conflict between airlines and passengers caused by the flight delays is prominent.

The conflict between airlines and passengers caused by the flight delays is prominent and some contradictions are intensified, then evolved into quarrels, fights even destroying public facilities, denied boarding, refusing to deplane and other extreme group events. According to the statistics of a south medium-sized airports, there were 33 group events caused by flight delays in 2002 and 41 group events in 2005.The number was increased to 94 in 2007.This series of events has seriously affected the image of the civil aviation 
industry and social harmony.

(3)Relying solely on the airlines, it is difficult to solve this problem.

Table1shows the proportion of reasons for flight delays in

China from 1998 to2010.According to statistics, airlines is the largest main responsibility for flight delays, but there are still about half of the flight delays caused by external factors beyond the control of airlines[4].

TABLE 1 Reasons for flight delays in China from1998

\begin{tabular}{|c|c|c|c|c|c|c|c|c|c|c|c|c|c|}
\hline & 1998 & 1999 & 2000 & 2001 & 2002 & 2003 & 2004 & 2005 & 2006 & 2007 & 2008 & 2009 & 2010 \\
\hline Airline & 67.5 & 70.4 & 73.5 & 75.9 & 77.5 & 79.0 & 70.9 & 65.7 & 44.2 & 45.9 & 45.82 & 42.72 & 47.0 \\
\hline Whether & 12.6 & 10.9 & 8.9 & 8.9 & 8.4 & 6.9 & 8.0 & 9.7 & 19.9 & 19.7 & 22.31 & 23.0 & 17.96 \\
\hline Airport & 1.5 & 1.5 & 1.7 & 1.4 & 1.6 & 2.6 & 3.0 & 3.1 & 2.0 & 0.9 & 2.35 & 1.21 & 0.82 \\
\hline ATC & 10.8 & 10.8 & 10.4 & 10.0 & 6.6 & 5.4 & 14.1 & 18.4 & 23.8 & 24.9 & 21.64 & 22.79 & 25.61 \\
\hline Passenger & 4.8 & 3.3 & 3.3 & 2.6 & 2.6 & 2.8 & 4.0 & 3.1 & 2.4 & 1.8 & 2.54 & 1.62 & 1.38 \\
\hline Others & 2.9 & 3.2 & 2.2 & 1.2 & 3.3 & 3.3 & 0.020 & 0.016 & 7.8 & 6.8 & 5.34 & 8.66 & 7.14 \\
\hline
\end{tabular}

Data sources: CAAC

(4) In current mechanism, it is impossible to solve the dispute caused by flight delays

Airlines is the largest governing subject for flight delays at the present stage in China. There are generally two departments responsible for handling flight delays: airline ground service and airport ground service. Since there is no real third-party ground services company, the airlines that don't set up ground service department at the airport would like to sign an agency with the airport that the airport ground service is responsible for dealing with the flight delays instead of the airlines.

In the first case that airline ground services department is responsible for handling flight delays, there are four corrupt practice. Firstly, not realizing that they are the main subject of liabilities, the airlines will blame the weather or other units, consciously or unconsciously. Secondly, the related personnel of the airlines often pass the buck each other. Thirdly, there is no professional management personnel of the airlines. Finally, as the other party in flight delays, the passengers do not believe information about delays provided by airlines. Therefore, relying solely on airline ground services department, it is difficult to deal with the flight delays efficiently. In second cases that airport ground service department or the third party service corporation dealing with the flight delays, there will be other corrupt practice besides the above four: for instance, the principal - agent problem.

Based on the above analysis, flight delay has become a public problem of the the aviation industry.

In 2008 June, the author conducted a survey about flight delays in 5 domestic airport. We collected valid questionnaires 246 copies of 1000 questionnaires. According to the results of the survey as Table 2 shows, a majority of people think that the flight delay is a public problem.

TABLE 2 Flight delays questionnaire

\begin{tabular}{|c|c|c|}
\hline Properties of flight delays & number/people proportion/\% \\
\hline $\begin{array}{c}\text { The airline's own problem, should be } \\
\text { solved by themselves }\end{array}$ & 97 & 39 \\
\hline $\begin{array}{c}\text { Public problems, need to solved by the } \\
\text { whole industry }\end{array}$ & 134 & 55 \\
\hline Not clear & 15 & 6 \\
\hline
\end{tabular}

\section{Flight Delays Disputes Governance Mechanism Innovation}

As its own mistakes, flight delays should be resolved by the airlines. As a public issue, governance of disputes caused by flight delays the goal of which is to provide fast and convenient air service, has obvious service characteristics. It can be solved by the government or he private sector ${ }^{[5]}$ The author proposes two new institutional design to solve this problem: one is setting up sectoral governance institutions of flight delays+mechanism of economic compensation reserve fund of flight delays; the other is introducing the third-party governance corporation of flight delays+flight delays economic compensation reserve system.

\subsection{Sectoral governance institutions of flight delays}

To solve this problem of flight delays --the public issues of the civil aviation industry, the government-led, multiply sectoral participation governance institutions must be established. This mechanism can be unified deployment of forces, the coordination of resources. There are long-term retention of FAA seats in the operation control center of Dallas and other large airport. FAA, the airport, the airline will cooperate once the serious flight delay occurred and this mechanism is very effective. Like the mechanism above, the sectoral governance institutions of flight delays should be set to the daily work mechanism the basic responsibility of which is to determine the cause of delay, release information, provide service, provide dispute resolution programme, distribute delay compensation etc.

Standing in the objective position, the authority determines and promulgates the reason of flight delay. This mechanism can guarantee the management of flight delay impartiality, fairness and systematicness and solve the conflict of flight delays quickly .

\subsection{Third-party governance corporation of flight delays}

The current trend to solve the public service problems is the introduction of the private sector and the implementation of third-party settlement mechanism. The third-party governance corporation of flight delays provides airlinescommissioned flight delays remedial services, such as advance warning, releasing information etc. The government authorized the company to determine delays responsibility, distribute 
economic compensation etc. The government can monitor and evaluate their work.

This mechanism can avoid the direct opposite of consumers and airlines, and can guarantee the efficiency of delay service.

\subsection{Comparisons between the two mechanism}

The two kinds of mechanism of each has its advantages and disadvantages. Industry management mechanism should be set up in the large congested airport only because of its high cost. While, the majority of the airport, as long as there is a certain delay rate, can introduce the third-party governance corporation of flight delays, because of low cost and higher operating efficiency. From the perspective of social division of labor and specialization, special governance corporation of flight delays will be a development trend in the future. Obviously, in the small and medium-sized airport, it is enough to solve the flight delays by the airline and airport.

\subsection{Financial security of flight delays disputes governance mechanisms}

There must be certain funds to run the agency, compensate delayed passengers both in industry management mechanism and third-party governance corporation of flight delays. For this reason, we think that it is necessary to establish economic compensation reserve for flight delays. The funds collected from airlines, airports, would be stored in a special account and used to compensate delayed passengers. Thus, this system can effectively reduce the flight delay disputes and resolve contradictions.

\subsubsection{Necessity of economic compensation reserve fund of flight delays}

we can effectively reduce the flight delay disputes and resolve contradictions based on the following four reasons: (1)Passengers can get compensation directly from economic compensation reserve fund of flight delays once the scope and the amount of compensation confirmed. (2) This mechanisms can avoid the main responsibility to refuse to pay compensation. (3)As the reserve fund is charged according to the proportion of annual delay rate, this mechanisms can strengthen their consciousness of reducing flight delays.(4) Delay governance department can withdraw the running cost from the reserve fund and the delay management company can get a certain profit.

\subsubsection{Overall mechanism}

(1)Sources of funds

Reserves is mainly collected from airlines and airports. ATC flow control can also cause flight delays, however it is necessary for aviation safety. Therefore, ATC should not be in the range of reserve collection.

(2) Form of collection

The reserve fund is charged according to the proportion of annual delay rate.

(3) The principle of collection

1. the principle of economic efficiency. 2. the principle of sustainability. 3. fairness principle
(4) The use of reserve

Stored into a special account, the reserve is arranged by. A certain proportion of the reserve fund is used for daily operation, all the other for compensation to passengers.

(5) Supervision of the fund

Reserve management institutions report the usage information regularly. The independent institutions are responsible for audit and supervision institutions responsible for the supervision. Fig.1 shows the overall mechanism of flight delays compensation reserve.

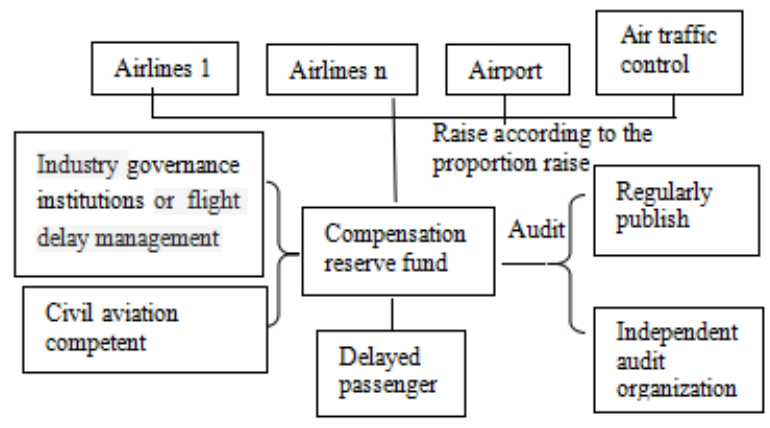

Fig.1 the overall mechanism of flight delays compensation reserve.

\subsubsection{The calculation method and steps}

The calculation method and steps of he reserve are as follows: Step 1:All passengers compensation at last year divided by the total number of flights of the airport makes the average amount of compensation for each flight. The average amount of compensation for each flight multiplied by the planned flight number, then multiplied by delay rate of the last year, the results plus the operating cost makes the amount of the reserve fund that airlines should hand in.

$$
\begin{array}{r}
K^{1}=H^{1}=\left(a_{1} / M_{1}^{0} * M_{1}^{1}+a_{2} / M_{2}^{0} * M_{2}^{1}+\right. \\
\left.\ldots a_{n} / M_{n}^{0} * M_{n}^{1}\right)+w^{1} * \frac{\alpha}{\alpha+\beta} \\
\alpha=\left(\alpha_{1}+\alpha_{2}+\alpha_{3}+\alpha_{4}+\alpha_{5}\right) / 5 \\
\beta=\left(\beta_{1}+\beta_{2}+\beta_{3}+\beta_{4}+\beta_{5}\right) / 5
\end{array}
$$

$H^{1}$ represents the number of the reserves fund of the first year that airlines should hand in. $K^{1}$ represents the number that airlines actually hand in. an represents the total compensation to passengers of the airlines $n$ in the previous year. $w^{1}$ represents the daily management costs of the first year. $M_{i}^{0}$ represents the total flights of the airlines $i$ in the previous year. $M_{i}^{1}$ represents the total flights of the airlines $i$ in this year. $\partial$ represents the average rate of the flight delays caused by the airlines in the previous five years. $\beta$ represents the average rate of the flight delays caused by the airport in the previous five years.

Step 2:According to reason for flight delays in the previous five years, we can figure out ratio of the airlines and the airport of the reasons for flight delays and then figure out of the reserves fund that the airport should hand in. of the reserves fund of the first year that airlines actually handed in. 


$$
L^{1}=F^{1}=H^{1} \times \frac{\beta}{\alpha}
$$

$F^{1}$ represents the number of the reserves fund of the first year that the airport should hand in. $L^{1}$ represents the number of the reserves fund of the first year that airlines actually handed in.

Step 3: We can figure out the reserves fund each airlines should hand in. The total compensation to passengers of the airlines $i$ in the first year divided by the total number of flights of the airport in last year, then multiplied by the flights number in this year ,the results plus the operating cost makes the amount of the first year reserve fund that airlines $i$ should hand in .

$$
\begin{gathered}
\mathrm{K}_{\mathrm{i}}^{1}=h_{i}^{1}=a_{i} / M_{i}^{0} * M_{i}^{1}+w_{1} * \frac{\alpha}{\alpha+\beta} * \\
\frac{a_{i} / M_{i}^{0} * M_{i}^{1}}{a_{1} / M_{1}^{0} * M_{1}^{1}+a_{2} / M_{2}^{0} * M_{2}^{1}+\cdots a_{n} / M_{n}^{0} * M_{n}^{1}}
\end{gathered}
$$

$T_{i}$ represents the time of the flight delays caused by the airlines i. $h_{i}^{1}$ represents the number of the reserves fund of the first year that airlines i should hand in. $k_{i}^{1}$ represents the number of the reserves fund of the first year that the airlines $i$ actually handed in.

Similarly, we can also figure out the reserves fund of the first year each airport should hand in. The ratio of the delayed time caused by the airport $i$ to all the delayed time caused by the airports multiplied by the number of the reserves fund that all the airports handed in.

$$
\begin{gathered}
l_{i}^{1}=f_{i}^{1}=\frac{s_{i}}{\sum_{i=1}^{n} s_{i}} * F^{1} \\
s_{i}=\sum_{j=1}^{m}\left(q_{i j} * s_{i j}\right)
\end{gathered}
$$

$S_{i}$ represents the time of the flight delays caused by the airport i. $f_{i}^{1}$ represents the number of the reserves fund of the first year that airport i should hand in. $l_{i}^{1}$ represents the number of the reserves fund of the first year that the airlines $i$ actually handed in. $q_{i j}$ represents the delayed passengers number in the delayed flight $j$ of the airport i.sij represents the delayed time in the delayed flight $\mathrm{j}$ of the airport $\mathrm{i}$.

Step 4: In the end of the first year, if there is balance and deficiency after deducting the daily operation expenses and compensation, the airlines or the airport can carry it forward to next year. The calculating method of the reserves fund in the next year is the same as the calculating method in the first year. Therefore, for the company which has balance, the the reserves fund in the next year the company should hand in minus the balance makes the amount the company actually hands in. While, for the company which has deficiency, the reserves fund in the next year the company should hand in plus the deficiency makes the amount the company actually hands in.

$$
\begin{aligned}
& k_{i}^{n}=h_{i}^{n} \pm c_{i}^{n-1} \\
& l_{i}^{n}=f_{i}^{n} \pm d_{i}^{n-1}
\end{aligned}
$$

$h_{i}^{n}$ represents the reserves fund of the $\mathrm{n}$ year that the airlines $\mathrm{i}$ should hand in.$k_{i}^{n}$ represents the reserves fund of the $\mathrm{n}$ year that the airlines $\mathrm{i}$ actually handed in. $f_{i}^{n}$ represents the reserves fund of the $\mathrm{n}$ year that the airport i should hand in. $l_{i}^{n}$ represents the reserves fund of the $\mathrm{n}$ year that the airport $\mathrm{i}$ actually handed in. $c_{i}^{n-1}$ and $d_{i}^{n-1}$ represent the deficiency or the balance of the airlines $i$ and airport $i$ in the $n-1$ year .

\subsubsection{Supervision framework}

First of all, the institutions for reserves fund supervision and control must clarify responsibilities through legislation. Secondly, take measures to regulate the reserve plan, ensure the budget and budget control independent. Finally, there must be long-term plans and measures to verify the reserve collection channels. Belonging to the government reserve in nature. the flight delay compensation reserve fund should be included in the national budget. The supervision framework for the flight delay compensation reserve fund mainly includes two levels. One is supervision from competent authority of the reserve, which is the highest level of rigid supervision, mainly be responsible for the examination and approval of the establishment of the reserve mechanism and the daily supervision. The other is the internal control mechanism, which is divided into internal control and supervision of reserve management of investor, is the basis level for regulation of reserves.

Flight delays is the public Issues of China's civil aviation industry, which is an important index of civil aviation service quality and the "bottleneck" of civil aviation and social harmonious development. Mobilizing the whole industry strength, even outside the power industry is necessary to solve the problem of flight delay. Industry management coordination mechanism should be set up in the airport in which the flight delays occur frequently; In more serious flight delays and medium-sized airports, flight delays management company should be introduced. While, the airlines or the airport can dispose the flight delays in the small airports. Only joint efforts of all forces, we can in reduce delays and resolve disputes.

\section{References}

[1] Zhu Weimin. Theory of organization and organizational innovation research. Business Economics and management, 2006 (1): 35 - 39

[2] Michael. Mcginnis. Multi center system and local public economic. Mao Shoulong, Li Mei, trans. Shanghai: Shanghai Sanlian bookstore, 2000: 99-100

[3] Li Xiong, Liu Guangcai, Yan Mingchi, et al. Caused economic losses of airlines and passengers of flight delay. Systems engineering, 2007 (11): 65- 67

[4] Zhang Dongqing, Ning Xuanxi. Considering the factors of income of travel time value model research system engineering, 2006 (21): 34 - 36 Rogene A Buchholz, William D Evans, Robert A W agley. Manage Ment response to pubic issues: concepts and cases in strategy formulation. London: Print ice $\mathrm{H}$ al, 1 1985: 1, 6- 8. 